\title{
A reabilitação psicossocial na atenção aos transtornos associados ao consumo de álcool e outras drogas: uma estratégia possível?
}

\section{The psychosocial rehabilitation of individuals with alcohol and drug use disorders: a possible strategy?}

\author{
Paula Hayasi Pinho ${ }^{1}$, Márcia Aparecida de Oliveira ${ }^{2}$, Marilia Mastrocolla de Almeida ${ }^{3}$ \\ ' Psicóloga, pós-graduanda da Escola de Enfermagem da Universidade de São Paulo (EEUSP). \\ 2 Professora doutora do Departamento de Enfermagem Materno-infantil e Psiquiátrica da USP. Coordenadora do Grupo de Pesquisa sobre Álcool e Drogas (GEAD) do \\ Departamento de Enfermagem Materno-infantil e Psiquiátrica da USP. \\ ${ }^{3}$ Terapeuta ocupacional, pós-graduanda da Escola de Enfermagem da USP.
}

Recebido: 26/09/2007 - Aceito: 20/02/2008

\section{Resumo}

Contexto: O consumo de álcool e drogas constitui-se em uma problemática mundial. A exclusão e a ausência de políticas específicas que marcaram a saúde pública brasileira demonstram a necessidade de reversão dos modelos assistenciais para contemplar as reais necessidades dos usuários que apresentam transtornos decorrentes do consumo de álcool e drogas. O desafio de formular uma política específica de prevenção, tratamento e reabilitação numa lógica que permita a singularidade e a reinserção social dos indivíduos tem sido tema dos diversos estudos nessa área. Objetivos: Identificar a utilização e compreensão do conceito da reabilitação psicossocial na atenção aos problemas associados ao consumo de álcool e drogas. Métodos: Revisão bibliográfica do banco de dados MEDLINE, de 1997 a 2007, sobre a reabilitação psicossocial no campo da atenção aos transtornos decorrentes do consumo de álcool e outras drogas. Resultados: Foram selecionados dez artigos que apresentavam similaridade com o tema. Conclusões: Encontramos os termos "suporte social" e "apoio social”, que fazem referência à importância da rede social na recuperação do usuário de álcool e outras drogas, assim como a reabilitação psicossocial determina a importância da rede social do indivíduo como um de seus eixos.

Pinho, P.H. et al. / Rev. Psiq. Clín 35, supl 1; 82-88, 2008

Palavras-chave: Reabilitação psicossocial, álcool, drogas.

\begin{abstract}
Context: The consumption of alcohol and drugs constitutes a worldwide problem. Exclusion and lack of specific policies for those who suffer with drug and alcohol dependency has marked Brazilian public health policy and demonstrated the need for change in the service models in order to contemplate the real needs of those with alcohol and drug abuse disorders. The challenge of formulating a specific prevention, treatment and rehabilitation policy within a framework that considers individuality and allows social reinsertion of individuals has been the subject of numerous studies in this area. Objectives: To identify the use and understanding of the concept of psychosocial rehabilitation associated with the problems of alcohol and drug abuse. Methods: Literature review in the MEDLINE database between 1997 and 2007 on psychosocial rehabilitation, specifically in the cases of alcohol and drug use disorders. Results: Ten articles were selected based on their pertinence to the subject. Conclusions: We found the term "social support" which refers to the importance of a social network to help the patient recovering from alcohol and drug addiction, just as the psychosocial rehabilitation - which determines the importance of the individual's social network - is one of its bases.
\end{abstract}

Pinho, P.H. et al. / Rev. Psiq. Clín 35, supl 1; 82-88, 2008

Key-words: Rehabilitation psychosocial, alcohol, drugs.

Instituição: Departamento de Enfermagem Materno-infantil e Psiquiátrica da Escola de Enfermagem da Universidade de São Paulo (EEUSP).

Endereço para correspondência: Paula Hayasi Pinho. Rua Artur Prado, 75, Bela Vista - 01322-000 - São Paulo, SP. E-mail: phpinho@terra.com.br 


\section{Introdução}

O consumo de álcool e outras drogas está inserido no cotidiano de grande parte da população mundial. Tal realidade está associada a uma série de outras situações de risco à saúde e vem sendo observada em diferentes países, em todos os continentes.

De acordo com a Organização Mundial da Saúde, cerca de $10 \%$ das populações dos centros urbanos de todo o mundo consomem abusivamente substâncias psicoativas, independentemente da idade, sexo, nível de instrução e poder aquisitivo. Salvo variações sem repercussão epidemiológica significativa, essa realidade encontra equivalência em território brasileiro (Ministério da Saúde, 2003).

Um estudo capitaneado pela Universidade de Harvard indica que, entre as dez doenças mais incapacitantes, cinco são de natureza psiquiátrica: depressão, transtorno afetivo-bipolar, alcoolismo, esquizofrenia e transtorno obsessivo-compulsivo (Ministério da Saúde, 2003).

Sobre o uso de substâncias psicoativas, o estudo de Harvard sobre a Carga Global da Doença trouxe a estimativa de que o álcool seria responsável por $1,5 \%$ de todas as mortes no mundo, bem como $2,5 \%$ do total de anos vividos com alguma incapacidade.

No Brasil, o II Levantamento Domiciliar sobre o Uso de Drogas Psicotrópicas, realizado em 2005 pelo Cebrid (Centro Brasileiro de Informações sobre Drogas Psicotrópicas), apontou que $12,3 \%$ das pessoas pesquisadas, com idades entre 12 e 65 anos, preenchem critérios para a dependência do álcool, e cerca de $75 \%$ já beberam pelo menos uma vez na vida (Carlini et al., 2007).

Segundo os dados da pesquisa, o uso na vida de álcool, nas 108 maiores cidades do país, foi de 74,6\%, porcentagem inferior a de outros países (Chile com 86,5\% e Estados Unidos com 82,4\%). Em todas as regiões, observaram-se mais dependentes de álcool do sexo masculino.

$\mathrm{O}$ uso na vida para qualquer droga (exceto tabaco e álcool) foi de $22,8 \%$. Essa porcentagem é, por exemplo, próxima ao Chile $(23,4 \%)$ e quase metade dos Estados Unidos $(45,8 \%)$.

O uso de solventes foi de $6,1 \%$, prevalência superior à verificada na Colômbia (1,4\%) e Espanha (4,0\%). Em contrapartida, a prevalência do uso na vida de solventes nos Estados Unidos foi de 9,5\%.

O uso na vida de maconha, nas 108 maiores cidades, foi de $8,8 \%$, resultado este próximo aos da Grécia $(8,9 \%)$ e da Polônia (7,7\%), porém abaixo dos Estados Unidos $(40,2 \%)$ e do Reino Unido (30,8\%).

A prevalência de uso na vida de cocaína nas 108 maiores cidades do país foi de $2,9 \%$, sendo próxima à da Alemanha (3,2\%), porém bem inferior a dos Estados Unidos (14,2\%) e do Chile (5,3\%). O uso na vida de crack foi de $1,5 \%$, cerca de duas vezes menor que no estudo norte-americano.
Os dados também indicam o consumo de álcool em faixas etárias cada vez mais precoces e sugerem a necessidade de revisão das medidas de controle, prevenção e tratamento.

A política de atenção integral a usuários de álcool e outras drogas

De acordo com o Ministério da Saúde (2007), até o ano de 2002 a saúde pública brasileira não vinha se ocupando devidamente com o grave problema da prevenção e tratamento dos transtornos associados ao consumo de álcool e outras drogas. Havia o predomínio de "alternativas de atenção" de caráter total, fechado, baseadas em práticas de natureza medicamentosa, disciplinar ou de cunho religioso-moral, reforçando o isolamento social e o estigma.

Somente a partir de 2003, o Ministério da Saúde formulou uma Política Nacional Específica para Álcool e Drogas, que assume o desafio de prevenir, tratar e reabilitar os usuários, segundo a Lei 10.216/01, marco legal da Reforma Psiquiátrica Brasileira. Os Centros de Atenção Psicossocial em Álcool e Drogas (CAPSad) passaram a ser considerados a principal estratégia de tratamento, bem como a estratégia de redução de danos; ambos tidos como ferramentas também nas ações de prevenção e promoção da saúde.

Esses dispositivos passaram a ser implantados, principalmente em grandes regiões metropolitanas com indicadores epidemiológicos relevantes, assim como várias experiências de manejo dos problemas de álcool e outras drogas na atenção básica, redes de suporte social, estrutura de atendimento hospitalar de urgência e emergência, e rede hospitalar de retaguarda para esses usuários. Atualmente, existem aproximadamente 160 CAPSad funcionando em todo o país, entretanto, essa rede ainda é insuficiente para atender à demanda cada vez mais crescente (Ministério da Saúde, 2007).

Os Centros de Atenção Psicossocial em Álcool e Drogas têm por finalidade prestar atendimento à população, em uma área de abrangência definida, oferecendo atividades terapêuticas e preventivas à comunidade.

Para o Ministério da Saúde, uma política de prevenção, tratamento e de educação para o uso de álcool e outras drogas necessariamente deve ser construída na interface de programas do Ministério da Saúde com outros ministérios, bem como com setores da sociedade civil organizada, especialmente na discussão sobre a restrição de propagandas de bebidas alcoólicas.

No ano de 2006 foi sancionada a nova lei sobre drogas no país (Lei 11.343/2006) que, embora não tenha abarcado toda a complexidade do tema, conseguiu avançar na garantia dos direitos com a supressão da pena de prisão para os usuários de drogas (Ministério da Saúde, 2007).

Em virtude da característica de heterogeneidade que predomina a dependência das drogas, uma vez que 
afeta as pessoas de distintas maneiras e por diferentes razões, nos mais diversos contextos e circunstâncias, uma política de atenção deve privilegiar as necessidades dos usuários, que, muitas vezes, não correspondem às expectativas dos profissionais de saúde com relação à abstinência, fator esse que dificulta a adesão ao tratamento, bem como as práticas preventivas ou de promoção voltadas aos usuários que não se sentem acolhidos em suas diferenças (Ministério da Saúde, 2004a).

Reconhecer o consumidor, suas características e necessidades, assim como as vias de administração de drogas, exige a busca de novas estratégias de contato e de vínculo com ele e seus familiares, para que se possa desenhar e implantar múltiplos programas de prevenção, educação, tratamento e promoção, adaptados às diferentes necessidades. Para que uma política de saúde seja coerente, eficaz e efetiva, deve-se levar em conta que as distintas estratégias são complementares e não concorrentes, e que, portanto, o retardo do consumo de drogas, a redução dos danos associada ao consumo e a superação do consumo, são elementos fundamentais para sua construção. (Ministério da Saúde, 2004a)

\section{Reforma psiquiátrica e reabilitação psicossocial}

A implantação e a regulamentação do Sistema Único de Saúde (SUS), instituído pela Constituição de 1988, configurado como "um conjunto de ações e serviços de saúde que tem por finalidade a promoção de maior qualidade de vida para toda a população brasileira”, tiveram como objetivo primordial a garantia do acesso de todos a uma assistência integral e eqüitativa à saúde. Nesse contexto, ocorreu uma reformulação profunda da assistência à saúde, visando à consolidação de uma rede de cuidados regionalizada, hierarquizada e integrada (Ministério da Saúde, 2004b).

No campo da assistência à saúde mental, desde a implantação do SUS até a aprovação da Lei 10.216 em 2001, inúmeras transformações das práticas e saberes foram desenvolvidas, garantindo aos usuários de serviços de saúde mental e, conseqüentemente, aos que sofrem por transtornos decorrentes do consumo de álcool e outras drogas a universalidade de acesso e direito à assistência, bem como à sua integralidade, valorizando a territorialização do atendimento, a partir da estruturação de serviços mais próximos do convívio social de seus usuários, configurando redes assistenciais mais adequadas às variadas demandas desse segmento da população, como assistência à saúde, benefícios sociais, esporte, lazer, cultura, moradia, trabalho, educação etc.

Atualmente, entende-se por Reforma Psiquiátrica um processo complexo, no qual quatro dimensões simultâneas se articulam e se retroalimentam.

Por um lado, pela dimensão epistemológica que opera uma revisão e reconstrução no campo teórico da ciência, da psiquiatria e da saúde mental, por ou- tro, na construção e invenção de novas estratégias e dispositivos de assistência e cuidado, tais como os centros de convivência, os núcleos e centros de atenção psicossocial, as cooperativas de trabalho, dentre outras. Na dimensão jurídico-política, temos a revisão de conceitos fundamentais na legislação civil, penal e sanitária (irresponsabilidade civil, periculosidade, etc.), e a transformação, na prática social e política, de conceitos como cidadania, direitos civis, sociais e humanos. Finalmente, na dimensão cultural, um conjunto muito amplo de iniciativas vai estimulando as pessoas a repensarem seus princípios, preconceitos e suas opiniões formadas sobre a loucura. É a transformação do imaginário social sobre a loucura, não como lugar de morte, de ausência e de falta, mas como também de desejo e de vida. (Amarante, 2004)

Assim sendo, tornou-se necessária a construção de uma rede de assistência baseada em dispositivos extrahospitalares de atenção psicossocial localizada no território e articulada a outros setores sociais, objetivando a reabilitação psicossocial e a reinserção social dos seus usuários que apresentem problemas decorrentes do uso de álcool e outras drogas. Tais dispositivos devem utilizar como referência a lógica ampliada de redução de danos, realizando uma procura ativa e sistemática das necessidades a serem atendidas, de forma integrada ao meio cultural e à comunidade em que estão inseridos e de acordo com os princípios da Reforma Psiquiátrica.

Segundo Saraceno (2001), a reabilitação é um processo que implica a abertura de espaços de negociação para o paciente, sua família e a comunidade circundante. Nesse sentido, introduz o conceito de contratualidade, ou seja, a capacidade de engendrar contatos sociais, que permitirão ao usuário subverter o processo de reclusão, que é resultado dos efeitos da doença mental e da exclusão social.

O conceito de reabilitação psicossocial propõe a ampliação da rede social, que envolve "profissionais e todos os atores do processo de saúde-doença, ou seja, todos os usuários e a comunidade inteira”. O processo de reabilitação consiste em "reconstrução, um exercício pleno de cidadania e, também, de plena contratualidade nos três grandes eixos: hábitat, rede social e trabalho com valor social" (Saraceno, 2001).

A reabilitação psicossocial também pode ser considerada um "processo pelo qual se facilita ao indivíduo com limitações a restauração no melhor nível possível de autonomia de suas funções na comunidade" (Saraceno, 2001).

De acordo com sua postulação, Saraceno (2001) demonstra a existência de uma estreita relação entre cidadania e saúde mental, posto que um indivíduo que não goze plenamente da cidadania é um risco para sua saúde mental, assim como um indivíduo que não goze plenamente de saúde mental estará impedido de exercer 
sua plena cidadania social. Nesse sentido, a questão central na reabilitação psicossocial está relacionada à elevação do sujeito de sua condição de doente mental para a condição de cidadão.

No contexto da Reforma Psiquiátrica e da desinstitucionalização, a reabilitação psicossocial deve procurar restituir a subjetividade do indivíduo na sua relação com a instituição, possibilitando a recuperação da contratualidade, ou seja, da posse de recursos para trocas sociais e, conseqüentemente, para a cidadania social (Saraceno, 2001). É nessa perspectiva que se insere a discussão da reabilitação psicossocial para os usuários de álcool e outras drogas, no sentido de desenvolver estratégias e ações intra-setoriais e intersetoriais efetivas, com o propósito de que estes possam assumir sua condição de sujeito social.

Com isso, o presente estudo tem por objetivo realizar uma revisão bibliográfica sobre a utilização da estratégia de reabilitação psicossocial no campo da atenção aos transtornos decorrentes do consumo de álcool e outras drogas.

\section{Metodologia}

Trata-se de um estudo de cunho bibliográfico que objetivou levantar a produção bibliográfica internacional (língua inglesa) sobre a utilização da estratégia de reabilitação psicossocial no campo da atenção aos transtornos decorrentes do consumo de álcool e outras drogas.

Realizou-se busca sistemática sobre o tema na base de dados MEDLINE, no período compreendido entre $1997 \mathrm{e}$ 2007. Para a busca, foram utilizados os seguintes descritores: "reabilitação psicossocial”, "álcool” e "drogas".

Foram encontrados 48 artigos; destes, 38 foram excluídos da amostra por não abordarem especificamente o tema em questão ou por terem sido publicados em outros idiomas. Assim, a amostra deste estudo constituiu-se de dez artigos, todos publicados entre os anos 2000 e 2007.

\section{Discussão dos resultados}

Os artigos analisados não utilizam o conceito de reabilitação psicossocial na prevenção ou tratamento de transtornos associados ao consumo de álcool e drogas. A maioria dos estudos utiliza o conceito de suporte/apoio social como fundamental ao tratamento de transtornos associados ao consumo de álcool e drogas. Outros tratam da relação entre "desordem mental” e consumo de álcool e drogas, demonstrando que indivíduos com desordem mental são prevalentes nos programas de tratamento para o abuso de substâncias (Compton et al., 2000; Havassy et al., 2004; Primm et al., 2000; Watkins et al., 2004).

Contrastando com o passado, quando tais indivíduos eram excluídos dos tratamentos para o abuso de substâncias, o número de indivíduos com desordem psiquiátrica vem aumentando e eles têm sido tratados nos programas de tratamento para o abuso de substâncias (McGovern et al., 2006). As pesquisas vêm demonstrando que a coocorrência do uso de substâncias e desordem mental faz com que existam baixas taxas no que se refere ao término do tratamento, à permanência no tratamento e às altas taxas de recaídas e re-hospitalização no póstratamento, se comparados com indivíduos que têm somente o problema do uso de substâncias (Compton et al., 2003; Weisner et al., 2003).

De acordo com os estudos internacionais, muitos indivíduos com desordens do uso de álcool e drogas têm co-ocorrência de desordens psiquiátricas (Helzer e Pryzbeck, 1988; Kessler et al., 1996). As taxas são particularmente altas entre aqueles que entram no tratamento da dependência química (Mertens et al., 2003; Wu et al., 2003), apresentando um grande desafio para as clínicas e para os planos de ação. Esses pacientes, na maioria das vezes, apresentam um curso mais crônico da doença (Kessler, 2004). Muitos estudos mostram piores resultados no tratamento para pacientes com duplo diagnóstico (Mos et al., 1994; Rounsaville et al., 1987; Weisner et al., 2000b; Zacny et al., 2003). Os efeitos da comorbidade psiquiátrica na dependência química são complexos e influenciados pelo início da desordem psiquiátrica relativa ao início da dependência química (Hasin et al., 2002).

Os estudos apontam que os serviços psiquiátricos raramente são incluídos no tratamento do dependente químico e, quando incluídos, são na maioria das vezes pobremente coordenados com o serviço de adicção (Edmund et al., 1997; Minkoff, 1997; Watkins et al., 2001). Inversamente, tais estudos sugerem que os serviços psiquiátricos também precisam melhorar os resultados para o tratamento do uso de álcool e drogas nos pacientes com comorbidade psiquiátrica (Drake et al., 1996; Hoff e Rosenheck, 1998), especialmente se integrados (Substance Abuse and Mental Health Services Administration, 2002). Estudos também têm descoberto que o tratamento psiquiátrico tem efeitos benéficos nos sintomas depressivos dessa população (Nunes e Levin, 2004; Pettinati, 2004; Torrense et al., 2005). No entanto, a importância da padronização dos serviços psiquiátricos (frequiência, intensidade, concentração de serviços) durante o tratamento da dependência química não tem sido examinada.

Nos Estados Unidos, os tratamentos que tratam a comorbidade psiquiátrica na dependência química têm recentemente se tornado uma importante política, porque muitos estados têm legalizado a paridade da saúde mental (p. ex.: seguros de saúde com cobertura para ocorrências psiquiátricas equivalentes àquelas coberturas para as condições médicas). Em 2000, a legislação da Califórnia (AB88) teve um mandato para a paridade do tratamento para esquizofrenia, desordens esquizoafetivas, depressão maior, transtorno bipolar, pânico, compulsão obsessiva, anorexia ou bulimia e desordens desenvolvidas por adultos. Atualmente, 33 
estados desse país têm na sua legislação a paridade da saúde mental (National Mental Health Association, 2004). Embora poucos estados tenham ampla cobertura, a maioria das leis cobre as desordens psiquiátricas severas identificadas na Califórnia.

Em resposta ao crescimento das necessidades de tratamento dessa população, têm-se desenvolvido padrões para "práticas melhores" e protocolos de tratamento para indivíduos com co-ocorrência de desordens (American Association of Community Psychiatrists, 2000; Drake et al., 2001; Mangrum et al., 2006; Minkoff, 2001).

As tentativas de aperfeiçoar o tratamento para indivíduos com co-ocorrência de desordens são extremamente complicadas em virtude da heterogenia dessa população, como características demográficas (gênero, etnia, idade), tipo de substância usada, tipo de desordem mental, incluindo todas essas combinações (Quello et al., 2005; Watkins et al., 2001).

Conforme descrito anteriormente, a heterogeneidade é de fundamental importância na problemática complexa do consumo de álcool e outras drogas, uma vez que afeta as pessoas de distintas maneiras e por diferentes razões. Nesse sentido, a estratégia da reabilitação psicossocial surge como uma resposta às diversas necessidades do usuário, respeitando sua singularidade, a partir da construção de um projeto terapêutico que esteja atento à ocorrência de uma ou mais desordens, pois conforme visto nos estudos analisados, o indivíduo que não tem diagnosticada a comorbidade psiquiátrica apresentará maiores dificuldades de adesão ao tratamento.

Fica evidente, segundo a literatura internacional, a necessidade de maior aprofundamento da relação entre transtornos mentais e consumo de substâncias psicoativas, no sentido de propor alternativas de atenção mais adequadas às necessidades dos usuários e na melhor adesão aos programas de prevenção e tratamento.

Outros estudos internacionais abordam o papel do suporte/apoio social no tratamento aos problemas relativos ao consumo de substâncias psicoativas, reconhecendo como uma influência positiva na saúde e na manutenção da saúde (Booth et al., 1992). O suporte social refere-se às informações recebidas por um indivíduo para que ele acredite que é importante, amado, estimado, valioso e que pertence a uma rede de comunicação e obrigação mútua (Coff, 1976). As relações interpessoais são consideradas importantes fatores de proteção contra os efeitos nocivos do estresse (Cohen e McKay, 1984).

O suporte social parece influenciar na motivação e nos resultados do tratamento para pacientes dependentes de drogas. Acerca do problema da dependência do álcool, Booth et al. (1992) sugerem a existência de influências externas como potencializadoras da autoconfiança dos indivíduos, ajudando-os na superação do problema do álcool.

A percepção da importância do suporte social oferecido pela família, amigos e outros participantes na recuperação dos usuários de drogas pode ter um papel vital na prevenção/desenvolvimento da recaída. $O$ suporte social está associado às necessidades subjetivas do indivíduo e também se relaciona à capacidade de suporte emocional da família e amigos na redução dos conflitos interpessoais e no estresse (Malhotra et al., 2001; McMahon, 2001). Isso também requer que o indivíduo assuma um papel ativo na estruturação benéfica do suporte social para ele mesmo, adotando outros caminhos, como deixar a companhia de amigos que usam drogas e aprendendo a recusar as ofertas para o uso (Malhotra et al., 2001).

Os estudos enfatizam que o suporte social no tratamento pode auxiliar na remoção de atritos e melhorar a interação interpessoal dos usuários e a sociedade. Isso pode $o$ ajudar a manter a abstinência e a assumir um novo papel social, como também o respeito perante a sociedade (Malhotra et al., 2001). Tal conceito aproximase da noção de reabilitação psicossocial como promoção da autonomia e da reinserção social dos indivíduos.

Em outro artigo analisado, o suporte social é considerado benéfico à saúde no impacto dos eventos adversos ou estressantes da vida (Cohenn e Wills, 1985). Diversos estudos têm documentado que o suporte social pode atuar como um fator protetor para o uso de drogas (Barrera et al., 1993; Frauenglass et al., 1997; Johnson e Pandina, 1991; McDonald e Towberman, 1993; Neaigus et al., 1994; Newcomb, 1997; Wills et al., 1992).

É atribuído um valor especial ao suporte dado pela família e amigos próximos aos usuários de álcool e drogas, indicando a importância de fortalecer e desenvolver a rede social na "libertação" das drogas, especialmente entre os pacientes que carecem dessa rede social ou necessitam dela para se fortalecerem.

Outro estudo (Fleeman, 1997) demonstra uma forte evidência de que o suporte social auxilia e efetiva $o$ tratamento ambulatorial e a desintoxicação residencial. Segundo esse autor, existem também outras vantagens, como a redução do estigma do paciente internado e o maior envolvimento da família

O suporte social na recuperação do uso de drogas é particularmente importante durante os estágios iniciais do tratamento, porque, nessa fase, o indivíduo tem maior probabilidade de ter sentimentos ambivalentes no que se refere a parar de consumir drogas (Laude $e t$ al., 2004).

O suporte comunitário é focado quase que exclusivamente nos familiares e amigos, com pouca atenção no potencial da religião ou da espiritualidade. Outro aspecto importante apontado é o maior envolvimento em atividades sociais, como trabalho, que acompanham e ajudam a definir a recuperação.

\section{Conclusão}

Embora a política de saúde brasileira no campo da atenção à saúde mental tenha como principal objetivo 
a desinstitucionalização e a reabilitação psicossocial do usuário que apresenta problemas decorrentes do consumo de álcool e outras drogas, na literatura internacional não foi encontrado nenhum estudo que se referisse à noção de reabilitação psicossocial nessa área.

Encontramos os termos "suporte social" e "apoio social”, que fazem referência à importância da rede social na recuperação do usuário de álcool e outras drogas, assim como a reabilitação psicossocial determina a importância da rede social do indivíduo como um de seus eixos.

Entretanto, o conceito de reabilitação psicossocial utilizado pela política de atenção nacional refere-se não apenas ao apoio ou suporte da família/amigos, ou seja, da rede social na prevenção e no tratamento, mas implica um conceito mais abrangente que está relacionado à elevação do sujeito de sua condição de doente para a condição de cidadão, subvertendo o processo de reclusão resultante dos efeitos da "doença" e da exclusão social (Saraceno, 2001).

\section{Referências}

Amarante PDC. - A (clínica) e a reforma psiquiátrica. In: Amarante PDC (org). Arquivos de Saúde Mental e Atenção Psicossocial. Rio de Janeiro: Nau; 2004.

American Association of Community Psychiatrists, 2000. - Principles for the care and treatment of persons with co-occurring psychiatric and substance disorders. Disponível em http://www.comm.psych.pitt.edu/ finds/dualdx.html.

A Política do Ministério da Saúde para Atenção Integral a Usuários de Álcool e Outras Drogas/Ministério da Saúde, Secretaria Executiva, secretaria de Atenção a Saúde, CN-DST/AIDS. - 1ํed. - Brasília: Ministério da Saúde, 2003.

Barrera, M.; Jr.; Chassin, L.; Rogosch, F. - Effects of social support and conflict on adolescent children of alcoholic and nonalcoholic fathers. Journal of Personality and Social Psychology, 64: 602-612, 1993.

Booth, B.M.; Russell. D.W.; Susan, S.; Laughlin, P.R. - Social support and outcome of alcoholism treatment: An exploratory Analysis. Am J Drug Alcohol Abuse 18: 87-101, 1992.

Carlini, E.A.; Galduróz, J.C.F.; Noto, A.R.; Nappo, S.A. - II Levantamento Domiciliar sobre o Uso de Drogas no Brasil: Estudo envolvendo as 108 maiores cidades do país - 2005. Brasília. Secretaria Nacional Antidrogas, 2007, p. 472.

Coff, S. - Social support as moderator of life stress. Psychosom Med 38: 300-314, 1976.

Cohen, S.; McKay, G. - Social support, stress and the buffering hypothesis: A theoretical Analysis. In: Baum, et al. editors, Handbook of Psychology and health, vol. IV. New Jersey: Erlbaum, p. 253-267, 1984.

Cohen, S.; Wills, T.A. - Stress, social support, and the buffering hypothesis. Psychologicak Bulletin, 98: 310-357, 1985.

Compton, W.M.; Cottler, L.B.; Ben Abdallah, A.; Phelps, D. L.; Spitznagel, E.L.; Horton, J.C. - Substance dependence and other psychiatric disorders among drug dependent subjects: race and gender correlates. Am J Addict 9: 113-125, 2000.

Compton, W.I.; Cottler, L.B.; Jacobs, J.L.; Bem-Abdallah, A.; Spitznagel, E.L. - The role of psychiatric disorders in predicting drug dependence treatment outcomes. Am J Psychiatry 160: 890-895, 2003.

Drake, R.E.; Essock, S.M.; Shaner, A.; Carey, K.B.; Minkoff, K.; Kola, L.; Lynde D.; Osher, F.C.; Clark, R.E.; Rickards, L. - Implementing dual diagnosis services for clients with severe mental illness. Psychiatr Serv 52: 469-476, 2001.

Drake, R.E.; Mueser, K.T.; Clark, R.E.; Wallach, M.A. - The course, treatment, and outcome of substance disorder in persons with severe mental illness. Am J Orthopsychiatry 66: 42-51, 1996.
Edmunds, M.; Frank, R.; Hogan, M.; McCarty, D.; Robinson-Beale, R.; Weisner, $C$ eds. - Managing Managed Care: Quality Improvement in Behavioral Health. National Academy Press, Washington, DC, 1997.

Fleeman, N.D. - Alcohol home detoxificacion. A literature review. Alcohol and Alcoholism 32: 649-656, 1997.

Frauenglass, S.; Routh, D.K.; Pantin, H.M.; Mason, C.A. - Family support decreases influence of deviant peers on Hispanic adolescents substance use. Journal of Clinical Child Psychology 26: 15-23, 1997.

Hasin, D.; Liu, X.; Nunes, E.; Samet, S.; Endicoot, J. - Effects of major depression on remission and relapse of substance dependence. Arch Gen Psychiatry 59: 375-380, 2002.

Havassy, B.E.; Alvidrez, J.; Owen, K.K.; - Comparisons of patients with comorbid psychiatric and substance use disorders: implications for treatment and service delivery. Am J Psychiatry 161: 139-145, 2004.

Helzer, J.E.; Pryzbeck, T.R. - The co-occurrence of alcoholism with other psychiatric disorders in the general population and its impact on treatment. J Stud Alcohol 49: 219-224, 1988.

Hoff, R.A.; Rosenheck, R.A. - Long-term patterns of service use and cost among patients wiyh both psychiatric and substance abuse disorders. Med Care 36: 835-843, 1998.

Johnson, V.; Pandina, R.J. - Effects of the family environment on adolescent substance use, delinquency, and coping styles. American Journal on Alcohol Abuse 17: 71-88, 1991.

Kessler, R.C.; Nelson, C.B.; McGonagle, K.A.; Edlund, M.J.; Frank, R.G.; Leaf, P.J. - The epidemiology of co-occuring addictive and mental disorders: implications for prevention and service utilization. Am J Orthopsychiatry 66: 17-31, 1996.

Kessler, R.C. - The epidemiology of dual diagnosis. Biol Psychiatry 56: 730-737, 2004.

Laudet, A.B.; Cleland, C.M.; Magura, S.; Vogel, H.S.; Knight, E.L. - Social support mediates the effects of dual-focus mutual aid groups on abstinence from substance use. Am J Community Psychol 34: 175-185, 2004.

Malhotra, A.; Malhotra, S.; Basu, D. - Social and cognitive approach in relapse prevention. Bengal J Psychiatry 10: 79-87, 2001.

Mangrum, L.F.; Spence, R.T.; Lopez, M. - Integrated versus parallel treatment of co-occurring psychiatric and substance use disorders. J Subst Abuse Treat 30: 79-84,2006.

McDonald, R.M.; Towberman, D.B. - Psychosocial correlates of adolescent grug involvement. Adolescence 28: 925-936, 1993.

McGovern, M.P.; Xie, H.; Segal, S.R.; Siembab, L.; Drake, R.E. - Addiciton treatment services and co-occurring disorders: prevalence estimates, treatment practices, and barriers. J Subst Abuse Treat 31: 267-275, 2006

McMahon, R.C. - Personality, stress and social support in cocaine relapse prediction. J Subst Abuse Treat 21: 77-87, 2001

Mertens, J.R.; Lu, Y.W.; Parthasarathy, S.; Moore, C.; Weisner, C.M. - Medi$\mathrm{cal}$ and psychiatric conditions of alcohol and drug treatment patients in an HMO: comparison to matched controls. Arch Intern Med 163: 2511-2517, 2003.

Ministério da Saúde. A Política do Ministério da Saúde para Atenção Integral a Usuários de Álcool e outras Drogas. Brasília: Ministério da Saúde, 2004a.

Ministério da Saúde. SAS. SAPE. Saúde Mental no SUS: os centros de atenção psicossocial. Brasília: Ministério da Saúde, 2004b.

Ministério da Saúde. Secretaria de Atenção à Saúde. Relatório de Gestão 2003-2006: saúde mental no SUS: acesso ao tratamento e mudança do modelo de atenção. Brasília: Ministério da Saúde, 2007.

Ministério da Saúde. Secretaria-Executiva. Subsecretaria de Assuntos Administrativos. Coordenação-Geral de Documentação e Informação. Centro Cultural da Saúde. Memória da loucura: apostila de monitoria. Brasília: Ministério da Saúde, 2004.

Minkoff K. - Best practices: developing standards of care for individuals with co-occurring psychiatric and substance use disorders. Psychiatr Serv 52: 597-599, 2001.

Minkoff, K. - Integration of Addiction and Psychiatric Services, in Public Sector Managed Mental Health Care: A survival Manual (Minkoff, K.; Pollack, D. eds). Harwood Academic Publishers, Amsterdam, the Netherlands, p. 233-245, 1997.

Moos, R.H.; Brennan, P.L.; Mertens, J.R. - Diagnostic subgroups and predictors of one-year readmission among late-middle-aged and older substance abuse patients. J Stud Alcohol 55: 173-183, 1994. 
National Mental Health Association. What have states done to ensure health insurance parity? Disponivel em: http://www.nmha.org/state/ parity/state_parity.cfm.

Neiagus, A.; Friedman, S.; Curtis, R.; Des Jarlais, D.; Furst, R.; Jose, B.; Mota, P.; Stephenson, B.; Sufian, M.; Ward, T. - The relevance of grug injectors sexual and risk networks for understanding and preventing HIV infection. Social Science and Medicine 38: 67-78, 1994.

Newcomb, M.D.; Bentler, P.M. - Impact of adolescent drug use and social support on problems of young adults: A longitudinal study. Journal of Abnormal Psychology 35: 64-75, 1988.

Newcomb, M.D - Psychosocial predictors and consequences of drug use: A developmental perspective within a prospective study. Journal of Addictive Diseases 16: 51-89, 1997.

Nunes, E.V.; Levin, F.R. - Treatment of depression in patients with alcohol or other drug dependence: a meta-analysis. JAMA 291: 1887-1896, 2004.

Pettinati, H.M. - Antidepressant treatment of co-occurring depression and alcohol dependence. Biol Psychiatry 56: 785-792, 2004.

Primm, A.B.; Gomez, M.B.; Tzolova-lontchev, I.; Perry, W.; Vu, H.T.; Crum, R.M. - Mental health versus substance abuse treatment programs for dually diagnosed patients. J Subst Abuse Treat 19(3): 285-290, 2000.

Quello, S.B.; Brady, K.T.; Sonne, S.C. - Mood disorders and substance use disorders: a complex comorbidity. Sci Prsct Perspect 3(1): 13-24, 2005.

Rounsaville, B.J.; Dolinsky, Z.S.; Babor, T.F.; Meyer, R.E. - Psychopathology as a predictor of treatment outcome in alcoholics. Arch Gen Psychiatry 44: 505-513, 1987.

Saraceno, B. Libertando identidades: da reabilitação psicossocial à cidadania possível. Belo Horizonte/Rio de Janeiro, Te Corá Editora/Instituto Franco Basaglia, 2001.

Substance Abuse and Mental Health Services Administration. Report to Congress on the Prevention and Treatment of co-occurring Substance
Abuse Disorders. Department of Health and Human Services, Bethesda, MD, 2002.

Torrens, M.; Fonseca, F.; Mateu, G.; Farre, M. - Efficacy of antidepressants in substance use disorders wiyh and without comorbid depression. A systematic review and meta-analysis. Drug Alcohol Depend 78: 1-22, 2005.

Watkins, K.E.; Burnam, A.; Kung, F.Y.; Paddock, S. - A national survey of care for persons with co-occurring mental and substance use disorders. Psychiatr Serv 52: 1062-1068, 2001.

Watkins, K.E.; Hunter, S.B.; Wenzel, S.L.; Tu, W.; Paddock, S.M.; Griffin, A.; Ebener, P. - Prevalence and characteristics of clients with co-occurring disorders in outpatient substance abuse treatment. Am J Drug Alcohol Abuse 30:749-764, 2004.

Weisner, C.; Matzger, H.; Kaskutas, L.A. - How important is treatment? Oneyear outcomes of treated and untreated alcohol dependent individuals. Addiction 98: 901-911, 2003.

Weisner, C.; Mertens, J.; Parthasarathy, S.; Moore, C.; Hunkeler, E.; Hu, T.W.; Selby, J.V. - The outcome and cost of alcohol and drug treatment in am HMO: day hospital versus traditional put patient regimens. Health Serv Res 35: 791-812, 2000

Wills, T.A.; Vaccaro, D.; McNamara, G. - The role of life events, family support, and competence in adolescent substance use: $A$ test of vulnerability and protective factors. American Journal of Community Psychology 20: 349-374, 1992.

Wu, L.T.; Ringwalt, C.L.; Williams, C.E. - Use of substance abuse treatment services by persons with mental health and substance use problems. Psychiatr Serv 54: 563-369, 2003.

Zacny, J.; Bigelow, G.; Compton, P.; Foley, K.; Iguchi, M.; Sannerud, C. College on problems of drug dependence taskforce on prescription opioid non-medical use and abuse: position statement. Drug Alcohol Depend 69: 215:232, 2003. 